\title{
The resonant dynamical evolution of small body orbits among giant planets
}

\author{
R. Gabryszewski ${ }^{1}$ and I. Włodarczyk ${ }^{2}$ \\ 1 Space Research Centre, ul. Bartycka 18A, 00-716 Warszawa, Poland \\ e-mail: r.gabryszewski@cbk. waw.pl \\ 2 Astronomical Observatory of the Chorzów Planetarium, WPKiW, 41-501 Chorzów, Poland \\ e-mail: astrobit@ka.onet.pl
}

Received 15 January 2003 / Accepted 25 April 2003

\begin{abstract}
Mean motion resonances (MMRs) can lead either to chaotic or regular motion. We report on a numerical experiment showing that even in one of the most chaotic regions of the Solar System - the region of the giant planets, there are numerous bands where MMRs can stabilize orbits of small bodies in a time span comparable to their lifetimes. Two types of temporary stabilization were observed: short period $\left(\sim 10^{4}\right.$ years $)$ when a body was in a MMR with only one planet and long period (over $10^{5}$ years) when a body is located in overlapping MMRs with two or three planets.

The experiment showed that the Main Belt region can be enriched by cometary material in its pre-active state due to temporary resonant interactions between small bodies and giant planets.
\end{abstract}

Key words. solar system: general - minor planets, asteroids - celestial mechanics

\section{Introduction}

Commensurabilities in a mean motion are very common in the Solar System. Resonant relations in the Main Belt have been known for nearly one and a half century - gaps in the distribution of asteroids were first linked to mean motion resonances (MMRs) by Kirkwood (1867). The influence of commensurabilities on the dynamics of small celestial bodies can be found in every region of the Solar System: from the Kuiper Belt (Nesvorny \& Roig 2000) to the vicinity of the Venus orbit (Milani \& Baccili 1999).

Resonant relations of small bodies in the region among giant planets have been studied for the last three decades. Long time orbital stability was found close to the $L_{4}$ and $L_{5}$ Lagrangian points of Jupiter (Everhart 1973), in the narrow bands centered on 7.02 and 7.54 AU (Franklin et al. 1989) and in the region between 24-27 AU (Holman 1997). Recent papers describe the local (Grazier et al. 1999) or global (Robutel \& Laskar 2001) dynamics in the region of the giant planets.

In the cited works the exploration of small body dynamics was used to find stable regions of the Solar System where particles can survive on Gyr timescales on circular or quasicircular orbits. However, the aim of this work is to investigate the dynamics of real particles in order to find the possible paths of their evolution and to learn about the role of MMRs as the mechanism of the orbital temporary stabilization process.

Send offprint requests to: $\mathrm{R}$. Gabryszewski, e-mail: r.gabryszewski@cbk.waw.pl

\section{Method of computations}

In most of the previous papers on the subject, the results were based on the integrations of the equations of motion for fictitious test particles, with initial orbital elements chosen adhoc. In this paper, on the contrary, we simulate the evolution of the orbital elements of real Centaurs and their clones. We chose orbits of all objects situated entirely inside the giant planets region: $1998 \mathrm{SG}_{35}, 1999 \mathrm{UG}_{5}, 2000 \mathrm{EC}_{98}, 2000 \mathrm{GM}_{137}$ and $2001 \mathrm{BL}_{41}$. All these objects are unnumbered, often singleoppositional which means that their elements stored in public databases can be approximated. That is why we decided to re-calculate their orbits using all available observations. Unfortunately, the data did not allow us to acquire the elements of $2000 \mathrm{GM}_{137}$ with a reasonable precision, so we omitted this object in our computations.

To compute the orbital elements of these real Centaurs at the initial epoch of our simulations, we took the observations from the files available at Minor Planet Center in Cambridge, USA. The sets of apparitions covered a very short time interval of about a month to a month and a half for the $1998 \mathrm{SG}_{35}, 2000 \mathrm{EC}_{98}$ and $2001 \mathrm{BL}_{41}$, and over 4 months for the $1999 \mathrm{UG}_{5}$. Observations were selected according to the mathematically objective criteria elaborated by Bielicki \& Sitarski (1991), assuming a normal distribution of their random errors. Only 1 observation of the $1998 \mathrm{SG}_{35}$ and 4 observations of the $1999 \mathrm{UG}_{5}$ were rejected, the rest were used in the orbit improvement process. Calculations were executed 
using Sitarski's orbital programme package (Sitarski 1971, 1979, 1984). Table 1 gives the elements acquired for the bodies.

\subsection{Particle cloning}

These small bodies move in the giant planets region, with the perihelion distance close to Jupiter orbit and with the aphelion between the orbits of Saturn and Uranus. Orbits of the particles are chaotic due to strong planetary perturbations, their Lyapunov time is short and lasts about 1000 years.

As an example, let us take two orbits of bodies moving in the gravitational field of the 4 planets - their 5 orbital elements are identical and they only differ in the starting semi-major axes by $1.5 \mathrm{~km}\left(10^{-8} \mathrm{AU}\right)$. Figure $1 \mathrm{~A}$ shows the eccentricity variations as a function of semi-major axis for two orbits - the nominal (crosses) and the changed one (circles) in a time span of only 11000 years. Figure 1B shows the logarithm of the divergence of semi-major axes between these orbits. The divergence is close to $10^{-8} \mathrm{AU}$ in the first 3000 years of the evolution but after this time it changes rapidly and both orbits start to evolve differently (see the huge leap of values of the logarithm in Fig. 1B). This means that even very small changes of one of the elements make the particle's dynamics quite different after a small time span. To avoid this problem, an ensemble of possible orbital elements is often used in such cases - the object's dynamical evolution is described in terms of probability.

Usually sets of elements are acquired by varying initial orbital elements within a reasonably small range, in most cases in the range of their mean errors. This procedure forms different orbital element sets but they cannot be treated as element sets of one celestial body. In fact they represent similar orbits of different bodies since these orbits do not fit the observations well. The sets of elements, used in the modelling and described in this paper, were generated in a different way. We used the method (found by Sitarski 1998) for creation of orbits which represent the observations well. This method makes it possible to produce any number of orbital element sets and all of them fit the observations. Hence, these orbits can be treated as possible orbits of one celestial body.

The dynamics of small bodies was modelled with the use of 404 orbital element sets created by the method mentioned above: for every real body 100 additional orbits were created. Table 2 shows the boundary values of all six elements for each real object.

\section{Numerical model}

Dynamical evolution was investigated by integrating a 6-body problem, with the central mass, 4 giant planets from Jupiter to Neptune, and a massless particle. We analyzed 404 sets of initial conditions, each of them consisting of positions and velocities for the planets (the same values in all cases) and for the massless particle. The orbital elements for the planets were taken from Bretagnon's planetary theory. For the massless particle, we used the 4 orbits of real Centaurs and 400 orbits cloned using Sitarski's method (100 clones for each real body). The initial positions and velocities were reduced to the barycentre of the inner Solar System. The sets were integrated separately
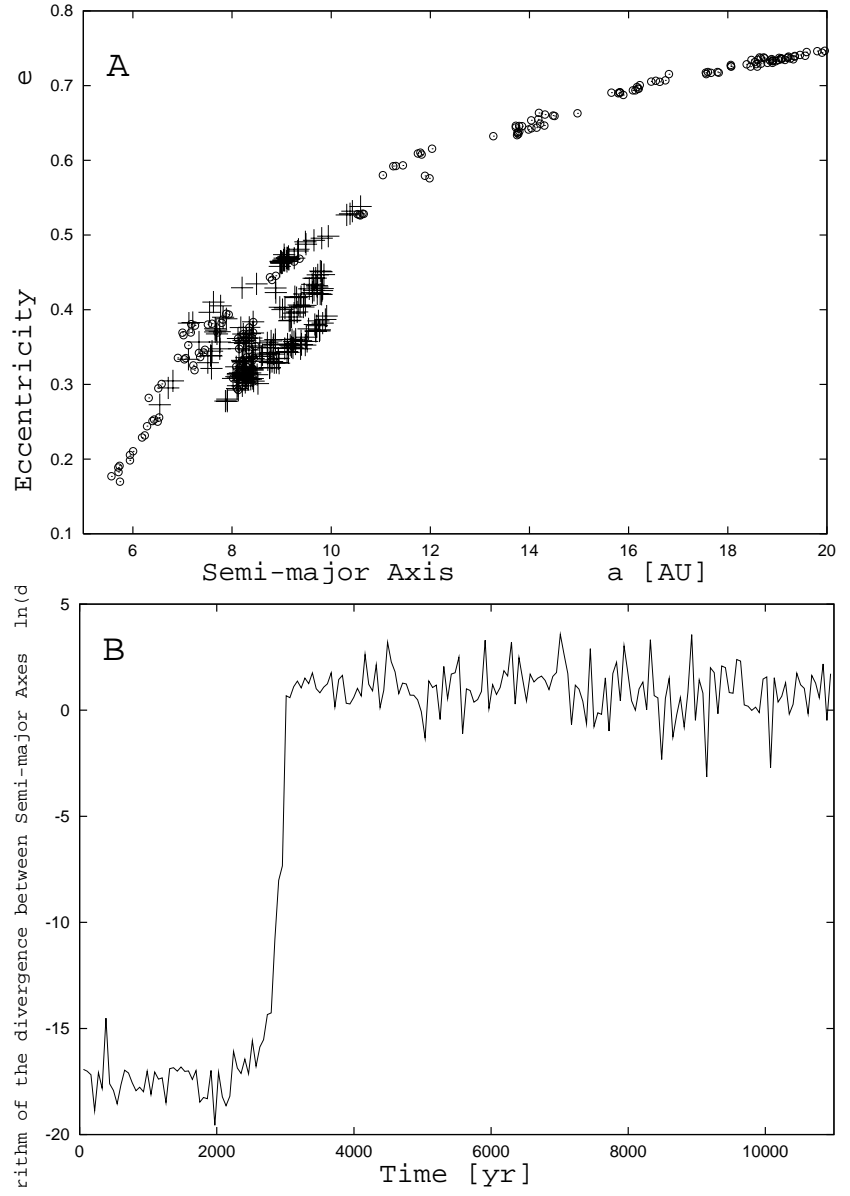

Fig. 1. A) The phase space of the eccentricity variations in a function of semi-major axis for two nearly identical orbits: the reference (crosses) and the changed one (circles). They differ only in the starting semi-major axes by a factor of $10^{-8}$. The integration time is $1.1 \times 10^{4}$ years. B) Time variations of the logarithm of the divergence in semi-major axes between orbits presented in the A). The leap near $T=3000$ is caused by two close encounters with Saturn of both objects.

200000 years forwards using the 15th order RADAU integrator (Everhart 1974, 1985) with an error tolerance set to $10^{-14}$. This integrator adjusts the step size to maintain the accuracy for all objects taking part in the process.

Locations of mean motion resonances were simply calculated with the use of Kepler's third law. The critical angle of the resonance was defined as follows:

$\sigma=k_{1} \cdot \lambda_{p}-k_{2} \cdot \lambda+\left(k_{2}-k_{1}\right) \cdot \tilde{\omega}$

where $k_{1}, k_{2}$ are integers, $\lambda_{p}$ and $\lambda$ are the mean longitudes of a planet and a test particle respectively, and $\tilde{\omega}$ denotes the longitude of the perihelion of test orbit. During the experiment only two-body MMRs were examined.

We accepted a special method for a resonance description. In our notation, a resonance $2 / 3$ means that a planet takes 2 revolutions around the barycentre in the time in which a small body takes 3 . 
Table 1. Orbital elements and their mean errors of the four bodies derived from the observational data on the epoch 19980706 ET.

\begin{tabular}{rccc}
\hline \hline Object & $q \pm \mathrm{d} q[\mathrm{AU}]$ & $e \pm \mathrm{d} e$ & $i \pm \mathrm{d} i[\mathrm{deg}]$ \\
$\mathrm{rms}[\mathrm{arcsec}]$ (number of obs.) & $\omega \pm \mathrm{d} \omega[\mathrm{deg}]$ & $\Omega \pm \mathrm{d} \Omega[\mathrm{deg}]$ & $T \pm \mathrm{d} T[\mathrm{ET}]$ \\
\hline $1998 \mathrm{SG}_{35}$ & $5.84069270 \pm 0.00028536$ & $0.30587190 \pm 0.00001753$ & $15.62341 \pm 0.00011$ \\
$0.38(49$ observations) & $337.90443 \pm 0.00385$ & $172.49261 \pm 0.00008$ & $20080210.31886 \pm 0.9552$ \\
\hline $1999 \mathrm{UG}_{5}$ & $7.46349029 \pm 0.00027588$ & $0.41436520 \pm 0.00006159$ & $5.58734 \pm 0.00006$ \\
$0.61(165$ observations) & $288.36650 \pm 0.01335$ & $87.02353 \pm 0.00058$ & $19981129.32162 \pm 0.21010$ \\
\hline $2000 \mathrm{EC}_{98}$ & $5.79188701 \pm 0.00393862$ & $0.45879307 \pm 0.00049997$ & $4.35728 \pm 0.00068$ \\
$0.48(38$ observations) & $163.21158 \pm 0.19737$ & $172.62330 \pm 0.00091$ & $20150505.81412 \pm 15.12784$ \\
\hline $2001 \mathrm{BL}_{41}$ & $6.84577522 \pm 0.21428445$ & $0.30172882 \pm 0.02988279$ & $12.50754 \pm 0.16842$ \\
0.49 (29 observations) & $131.62288 \pm 6.14676$ & $280.58723 \pm 0.18671$ & $19980120.61111 \pm 68.56299$ \\
\hline
\end{tabular}

Table 2. Dispersion of objects' orbital elements - boundary values chosen from the set of 100 additional orbits, separately for each single element.

\begin{tabular}{ccccccc}
\hline \hline Object & $T$ & $q$ & $e$ & $\omega$ & $\Omega$ & $i$ \\
\hline $1998 \mathrm{SG}_{35}$ & 20080210.55929 & 5.84125774 & 0.30592525 & 337.91370 & 172.49281 & 15.62380 \\
& 20080210.07866 & 5.83989244 & 0.30583258 & 337.89299 & 172.49240 & 15.62309 \\
\hline $1999 \mathrm{UG}_{5}$ & 19981129.85783 & 7.46422563 & 0.41450678 & 288.40122 & 87.02481 & 5.58749 \\
& 19981128.91046 & 7.46296851 & 0.41424401 & 288.34168 & 87.02197 & 5.58720 \\
\hline $2000 \mathrm{EC}_{98}$ & 20150612.06479 & 5.80080946 & 0.46019067 & 163.70350 & 172.62571 & 4.35859 \\
& 20150324.73689 & 5.78133382 & 0.45761563 & 162.65756 & 172.62160 & 4.35549 \\
\hline $2001 \mathrm{BL}_{41}$ & 19981118.53404 & 7.34292453 & 0.40165728 & 152.56526 & 281.04189 & 12.98724 \\
& 19971026.14988 & 6.23554911 & 0.24252707 & 119.25777 & 280.07133 & 12.10321 \\
\hline
\end{tabular}

\section{The influence of MMRs on small body dynamics}

The experiment showed that MMRs can temporarily stabilize numerous elliptic orbits in the Jupiter - Uranus region in periods of time comparable to their typical lifetimes (i.e., the time over which non-resonant particles can survive in that region).

Two types of resonant behaviour were observed. The first one - when a small body is in a two-body MMR with only one planet. This relation usually lasts a short time, from several hundred up to 35000 years. There were 120 particles of this resonant type. We only noticed two particles in a resonance with one planet for a longer time span.

One of these particles, corresponding to one of the $1998 S G_{35}$ clones, is shown in Fig. 2. After 118000 years of chaotic evolution the particle enters the $2 / 3$ resonance with Jupiter and remains in this relation to the end of the integration process. Figure $2 \mathrm{~A}$ presents variations of the semi-major axis as a function of time. The critical angle usually librates about $0^{\circ}$ when a body is located in this particular resonance (see Fig. 2B). Even high and growing amplitude (over $150^{\circ}$ ) of the librations is incapable of destabilizing the dynamics of the particles in a time span of over 80000 years.

Similarly to this Hilda type orbit, nearly all other orbits remaining in resonance with only one planet behave like the Toro class orbits (see Fig. 3). This class defined by Milani (1989) consists of Earth-crossing objects which avoid close approaches by entering a MMR. Most particles remaining in the resonant relation with Jupiter or Saturn cross their orbits in ecliptic projections but cannot approach them. The time of such resonant relations is smaller than for Toro like orbits (with some exceptions) but this can be caused by stronger perturbations acting on a body in the giant planet region.
The second type of resonant behaviour is characterized by much longer resonant interactions up to 160000 years in our sample. Test particles are in two-body MMRs with 2 or 3 planets (see Fig. 4). These overlapping resonant relations can stabilize orbits far more effectively - for over $10^{5}$ years. Figure 4 presents the evolution of the MMRs critical angles for one of these orbits. When two MMRs overlap each other, the behaviour of the critical angle of the dominant resonance starts to vary (see Fig. 4C) - the critical angle begins to circulate with an overlapped libration of amplitude almost constant. Irregularities of the rotation and of the amplitude of libration do not mean that the body will leave the MMRs in the near future but the possibility that a small body will approach a planet increases with time. Only 9 to $15 \%$ of orbits were stabilized on time spans longer than 35000 years (according to the object).

Figure 4 shows that overlapping resonances can also protect the body from close approaches to planets. The disturbing resonance does not necessarily destabilize the orbit of the body if the influence is weak.

\section{Migration to the main belt}

About $30-40 \%$ of test bodies migrate to the Main Belt on noncometary (asteroidal) orbits (see Hilda type orbit in Fig. 2). Most of them leave this region in a dynamically short time, usually in less than 250 orbital periods without entering a MMR, but about $30 \%$ of them will have resonant interactions with Jupiter and/or Saturn. When they enter a MMR, their lifetime in the Main Belt grows rapidly - up to 20000 orbital revolutions in our calculations.

The modelling shows that proto-cometary objects in their pre-active state can enrich the Main Belt population. A particularly interesting case is that of the short-lived resonant groups 

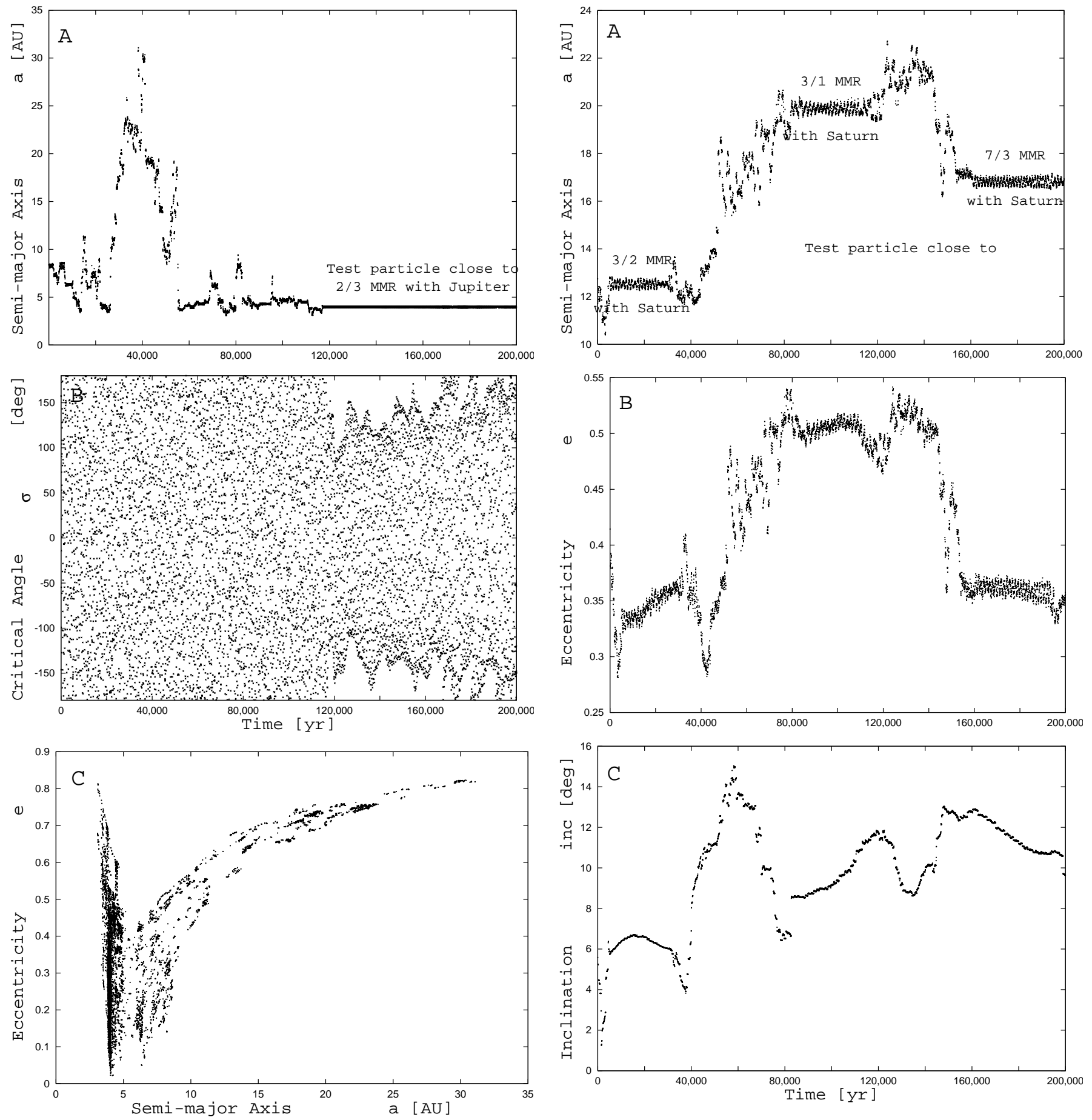

Fig. 2. $1998 \mathrm{SG}_{35}$ - test particle no 35. A) Variations of the semimajor axis as a function of time. The orbit is locked in a 2/3 MMR with Jupiter after 118000 years of dynamical evolution. B) Variations of the critical angle as a function of time. When the particle is in resonance, the critical angle starts to librate about $0^{\circ}$ with a large amplitude (dotted-free area indicates values of angles which are unaccessible for the librating body). The amplitude increases in time and the libration is hardly visible at the end of the integration (dotted-free area almost disappears). The particle will probably leave the resonance in several thousand years. When the body remains outside the resonance, values of the critical angle vary at random. C) The phase space of the eccentricity variations in the function of the semi-major axis. When a body is located in a resonance, the eccentricity can reach high values but the particle does not leave the MMR. The body seems to "stick" to the resonance.

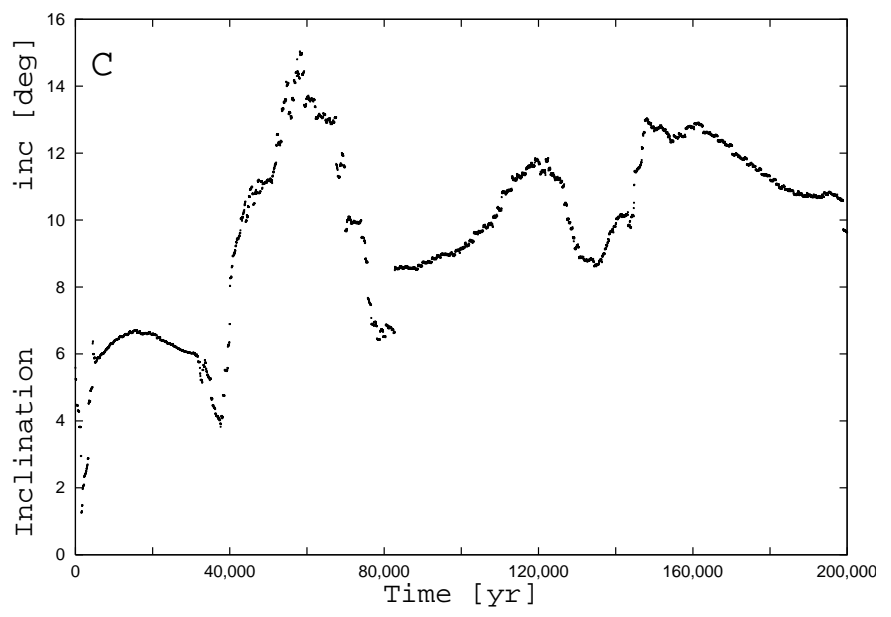

Fig. 3. $1999 \mathrm{UG}_{5}$ - test particle no 1. A) During its dynamical evolution, the particle enters different MMRs. When in resonance, the variations of eccentricity B) and inclination $\mathbf{C}$ ) values evolve slowly (but not periodically) in small boundaries due to the lack of close approaches to planets. Most of these orbits are Toro class, which means that they cross planetary orbits (in ecliptic projections) but avoid close approaches to these massive bodies.

of asteroids, like the Griquas. This is a group of resonant asteroids dynamically differentiated from the other resonant groups, and that cannot be primordial resonant bodies due to their short lifetimes. These vary from $10^{4}$ to $10^{7}$ years (Roig et al. 2002) which may indicate that a certain per cent of these asteroids 

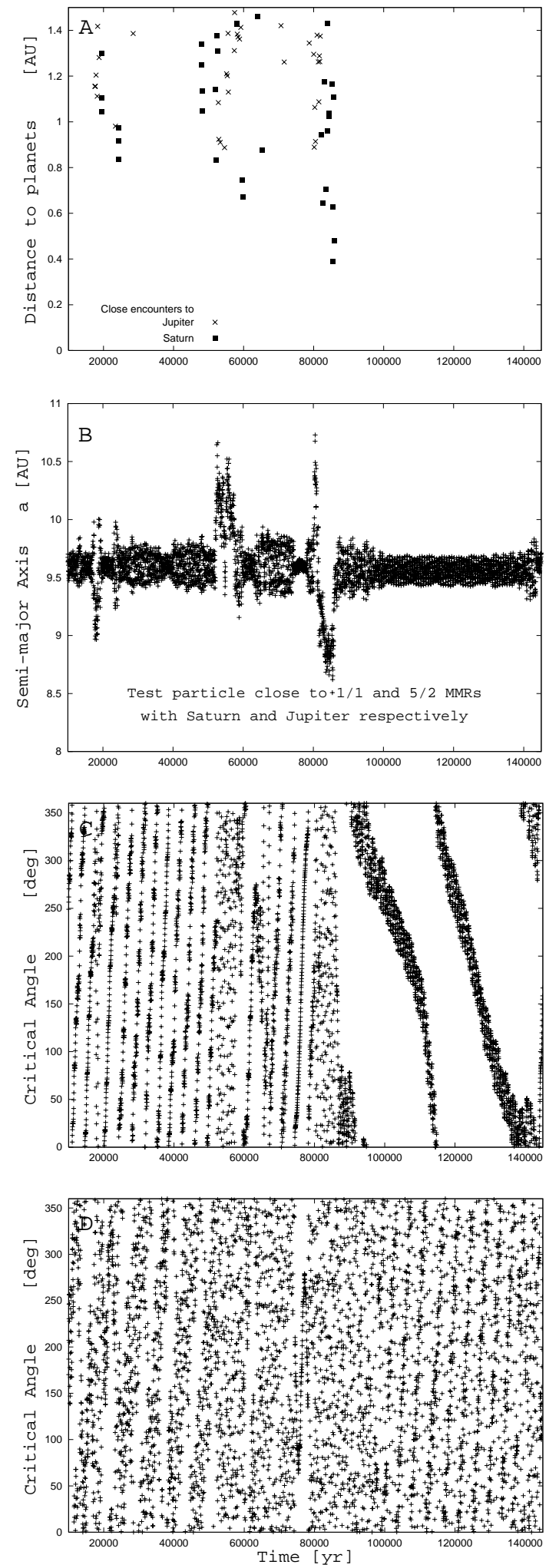

Fig. 4. $2001 \mathrm{BL}_{41}$ - test particle no 11. A) The distance between the body and planets as a function of time. B) Variations of the semi-major axis as a function of time. The orbit is located in overlapping MMRs due to near resonant relations between Jupiter and Saturn (so called Great Inequality). Time variations of the MMR critical angles of Saturn C) and Jupiter D) are also presented. After 100000 years of dynamical evolution, when the body remains in 1/1 MMR with Saturn and in 5/2 disturbing MMR with Jupiter, it has no close approaches to both planets. came from another regions. Have they come from the outer Solar System? Unfortunately, our integrations do not give an answer to this question. The calculations indicated only 1 particle with $3.1<a<3.27$ and $e>0.35$ close to a $1 / 2 \mathrm{MMR}$ with Jupiter with the orbital lifetime longer than $10^{4}$ years.

We decided to integrate backwards the equations of motion of 147 real asteroids moving close to $1 / 2$ MMR with Jupiter to find out if these objects are able to come from the region outside the orbit of Jupiter. The results shows that 1/2 MMR members can be enriched by Centaurs coming from two different regions but the backward simulations cannot be treated as the evidence.

\section{Islands of temporary stability}

The numerical experiment allowed one to find temporary "islands of stability" in the chaotic region of the giant planets bands where particles are trapped for the time estimated for $10^{4}-10^{5}$ years. These "islands" are observed near 6, 6.8, 8.2, $9.5,10,10.5,11,11.6,12.1,13,15.1$ and $17.3 \mathrm{AU}$ in the giant planets region, and also close to 2.8-3.1 and 3.4-4.2 AU in the Main Belt.

Figure 5 shows the bands of stability in the $(a, e)$ plane. All orbits located in these zones were temporarily stabilized by MMRs in the time span of over $10^{4}$ years. This period of time is comparable with the mean lifetime of the nonresonant particles in the region among giant planets estimated by Franklin et al. (1989). Most of the resonances are observed in the Main Belt and outside Saturn's orbit. The region between Jupiter and Saturn is weakly inhabited. This can be caused by strong gravitational perturbations from these two planets. The other hardly inhabited region (in fact nearly empty) is located outside the orbit of Uranus. This can be due to the ensemble selection.

The most effective MMRs stabilizing dynamics of particles are gathered in Table 3.

\section{Discussion and conclusions}

The region among the giant planets is one of the most chaotic in the Solar System. Strong perturbations cause small bodies to evolve in the region among giant planets over a dynamically short time equal to the $10^{3}-10^{4}$ years. Our results show that this time can be much longer if the body is temporarily trapped by a MMR with a planet.

Temporary stable orbits of the Centaurs seem to be a good example of the "resonance stickness" (Dvorak et al. 1998; Tsiganis et al. 2000). Gravitational perturbations can easily push a body into a MMR even if the eccentricity of the body is high. The evolution of the semi-major axis, eccentricity and inclination is chaotic in the borders of the resonance. The perturbations can push the body outside the resonance in timescale of $10^{4}-10^{5}$ years in the same way as they pushed the object into this MMR. The number of small bodies which enter a resonance on highly eccentric orbits during a short time of dynamical evolution (200000 years) indicates that a resonance sticking is a common mechanism affecting particle lifetimes in chaotic zones. 


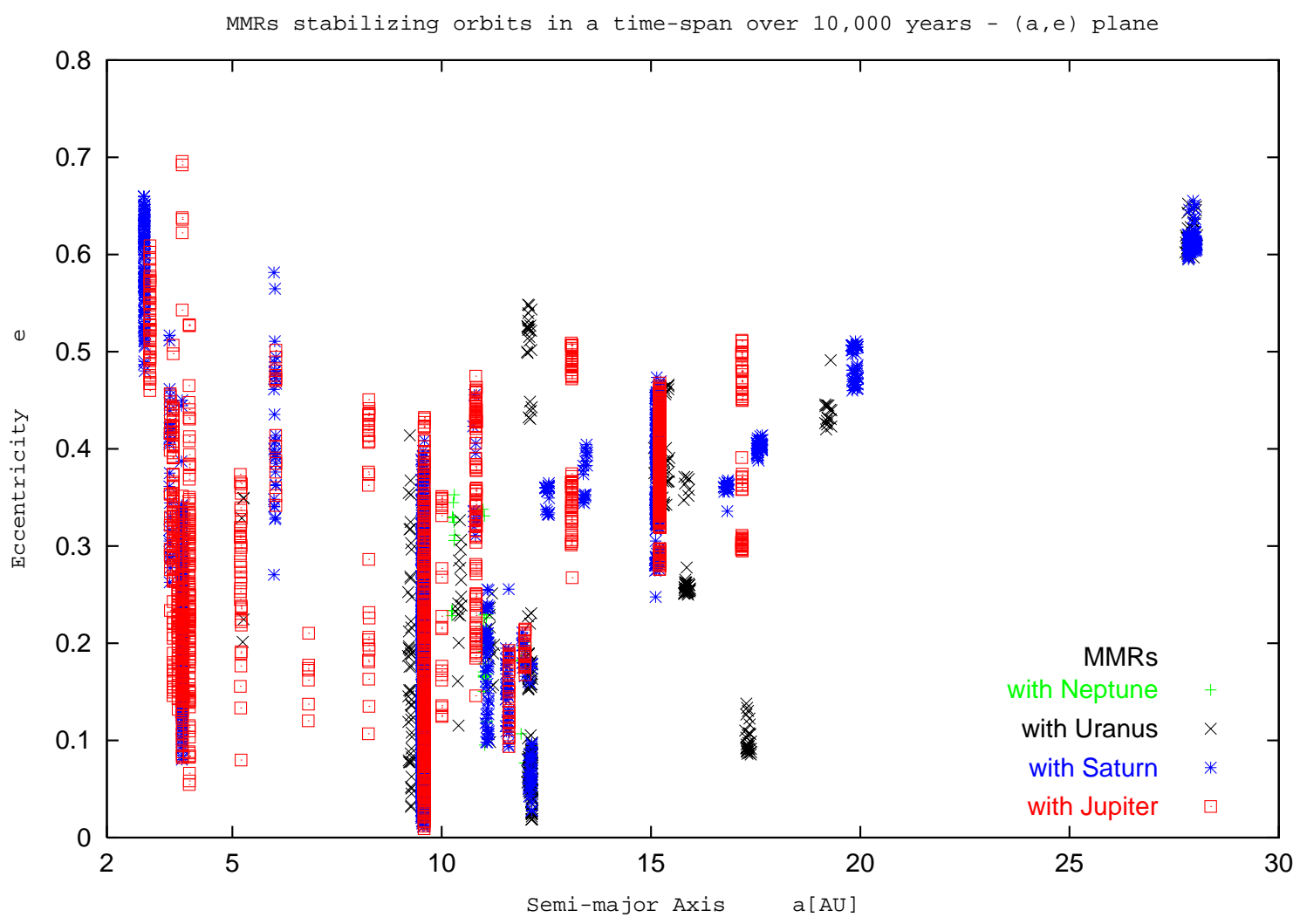

Fig. 5. "Islands of temporal stability" among the giant planets in the $(a, e)$ plane. High eccentricities do not prevent MMRs from stabilizing orbits in a time span longer than $10^{4}$ years due to stickness of resonances. The region outside Uranus seems to be empty but this can be caused by the ensemble selection. (This figure is available in color in the electronic version.)

To ensure that the phenomenon we encountered is in fact the resonance stickness, we investigated the autocorrelation function of the time series of the osculating elements of one of the resonant orbits. If the body is "stuck" to a resonance we observed quasi-periodic variations of orbital elements on a timescale much longer than the Lyapunov time of this orbit (Tsiganis et al. 2000).

Figure 6 presents the autocorrelation function of the semimajor axis as a function of the time-lag $k$ for one of the test particles being in $1 / 1$ and 5/2 MMRs with Saturn and Jupiter, respectively. Time series consisted of 1302 points (which corresponds to 40000 years) starting at $t=100000$ years. The series is strongly suppressed in time but this is due to the presence of gravitational perturbations from the giant planets in our 6 body problem. If we had a 3-body problem the suppression would be absent. Although the suppression is high, quasi-periodic variations can be observed. This seems to confirm the thesis of resonance stickness.

The most unintuitive result is that overlapping resonances can stabilize small body orbits on a much longer timescale than single resonances. The overlapping resonances are usually responsible for chaotic evolution, but our results show that they can stabilize dynamics rather than destabilize it. When we observe the particle motion in overlapping resonance with planets, we can see that its evolution is chaotic but only inside the borders of the MMRs. The resonances protect the body from close approaches to planets. Giant planets are close to resonant relations (Jupiter - Saturn - Uranus and Saturn - Uranus -
Table 3. Frequently observed MMRs and their stabilization time.

\begin{tabular}{ccl}
\hline \hline Object & MMR(s) & $\begin{array}{l}\text { time of orbital } \\
\text { stabilization }\end{array}$ \\
\hline $1998 \mathrm{SG}_{35}$ & $2 / 3(\mathrm{~J})$ & $80 \mathrm{kyrs}$ \\
& $2 / 1(\mathrm{~S})$ and $5 / 1(\mathrm{~J})$ & $50 \mathrm{kyrs}$ \\
& $1 / 1(\mathrm{~S})$ and $5 / 2(\mathrm{~J})$ & $50 \mathrm{kyrs}$ \\
& $6 / 7(\mathrm{U}), 1 / 3(\mathrm{U}), 4 / 9(\mathrm{~N})$ & $20 \mathrm{kyrs}$ \\
\hline $1999 \mathrm{UG}_{5}$ & $2 / 1(\mathrm{~S})$ and $5 / 1(\mathrm{~J})$ & $60 \mathrm{kyrs}$ \\
& $1 / 4(\mathrm{~S})$ and $5 / 8(\mathrm{~J})$ & $35 \mathrm{kyrs}$ \\
& $5 / 4(\mathrm{~S})$ and $2 / 9(\mathrm{~N})$ & $25 \mathrm{kyrs}$ \\
& $1 / 1(\mathrm{~S})$ and $5 / 2(\mathrm{~J})$ & $20 \mathrm{kyrs}$ \\
& $4 / 7(\mathrm{~J})$ & $10 \mathrm{kyrs}$ \\
\hline $2000 \mathrm{EC}_{98}$ & $1 / 4(\mathrm{~S})$ and $5 / 8(\mathrm{~J})$ & $120 \mathrm{kyrs}$ \\
& $5 / 2(\mathrm{~J}), 1 / 1(\mathrm{~S})$ and $1 / 3(\mathrm{U})$ & $110 \mathrm{kyrs}$ \\
& $4 / 9(\mathrm{~J})$ & $65 \mathrm{kyrs}$ \\
& $1 / 1(\mathrm{~S})$ and $5 / 2(\mathrm{~J})$ & $30 \mathrm{kyrs}$ \\
\hline $2001 \mathrm{BL}_{41}$ & $2 / 1(\mathrm{~S})$ and $5 / 1(\mathrm{~J})$ & $150 \mathrm{kyrs}$ \\
& $2 / 1(\mathrm{~S}), 5 / 1(\mathrm{~J})$ and $5 / 7(\mathrm{U})$ & $90 \mathrm{kyrs}$ \\
& $1 / 1(\mathrm{~S})$ and $5 / 2(\mathrm{~J})$ & $70 \mathrm{kyrs}$ \\
\hline
\end{tabular}

Neptune, Sussmann \& Wisdom 1988, 1992) which means that a small body can be locked in a MMR with 1 planet and close to MMRs with the other 1 or 2 massive bodies. When gravitational perturbations change the orbit of the particle, the body 


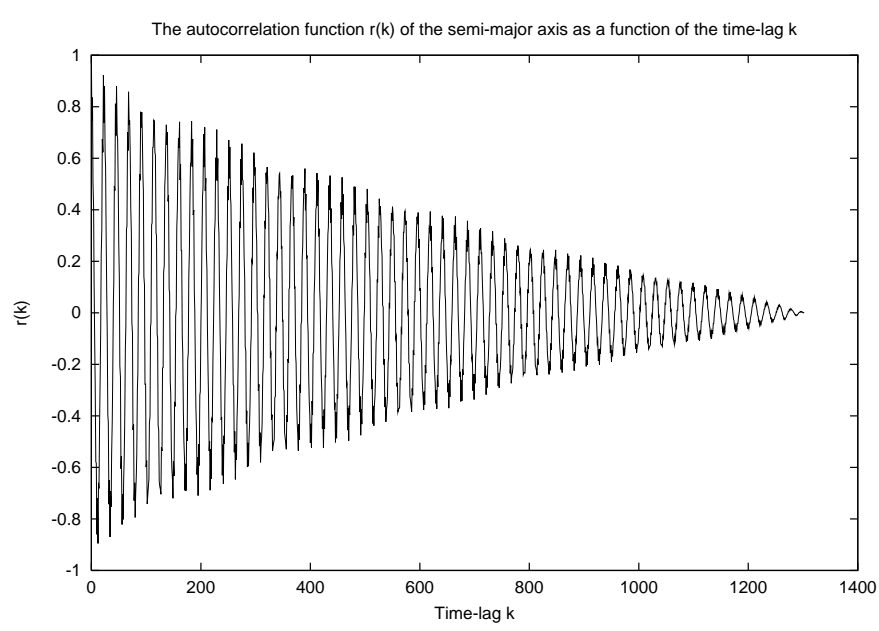

Fig. 6. $2001 \mathrm{BL}_{41}$ - test particle No. 11. The autocorrelation function $r(k)$ of the semi-major axis as a function of the time-lag $k$. Time series consists of 1302 points which correspond to 40000 years of time.

can be easily "pumped up" into another MMR. Phase space of MMRs is thicker when the eccentricity increases and hence bodies can remain in overlapping MMRs. The overlapping resonances protect the body from close approaches to planets (which are the main mechanism of chaos in the region) and according to our calculations bodies are unable to change the portion of the phase space where they evolve, on a timescale of over $10^{5}$ years.

Resonances between the test particle and the 4 planets have not been observed. This is in good agreement with Sussman's and Wisdom's works cited above.

The results presented in this work indicate that Centaurs may also enrich the population of asteroids in the Main Belt. MMRs can stabilize Centaur orbits on the timescale of $10^{4}-10^{5}$ years. This is a very short period of time in comparison with the lifetime of some asteroidal families in this region but shows that the Main Belt can include non-processed inactive cometary nuclei, not only extinct material. It is hard to say if such nuclei can be called primordial but our results show that the material can reach the asteroid belt on non-cometary orbits, without approaching the Sun. The particles did not enrich specific groups of asteroids in the Main Belt. The only group which seems to be overpopulated are Trojans.

We tried to consider if Centaurs can be a parental population of groups of asteroids with the lifetime comparable to $10^{4}-10^{5}$ years. Griquas are an example of such a group. They do not originate in the break-up event, a lot of the group members have lifetimes comparable to the lifetimes of Centaurs in the Main Belt. But, unfortunately, our modelling did not produce a group of objects with $3.1<a<3.27$ and $e>0.35$. Although, when we integrated backwards all known orbits of the Griqua group members, we found that some of them are able to come from the regions among giant planets. Further investigations are needed to explain the origin of Griqua type bodies.

The earlier works of Everhart (1973) and Franklin et al. (1989) indicate that there are two narrow bands at 7.02 and 7.54 AU where particles can survive longer than $10^{6}$ years. In
1997 Holman (1997) pointed to a region between 24 and 27 AU where low inclined $\left(i \leq 1^{\circ}\right)$ and low eccentric $(e \leq 0.01)$ objects can survive 1 Gyr. Our results do not indicate any stable orbits close to those bands. This means that the resonant dynamics is not the main mechanism responsible for stabilizing orbits in these areas. Slow evolution of low inclined, quasi circular orbits close to bands at 7.02 and 7.54 AU and in the Holman's region is caused by tiny variations of the gravitational force acting on bodies.

Centaurs are perceived as a intermediate stage between Kuiper Belt objects and Jupiter Family comets (Levison \& Duncan 1997). During the integration we noticed only $10 \%$ of orbits with $q<1.5 \mathrm{AU}$ and $Q>5.0 \mathrm{AU}$. Most of them were orbits of $1998 \mathrm{SG}_{35}$ and $2001 \mathrm{BL}_{41}$ objects (79 and 13 orbits respectively).

It is hard to compare our results with those presented in Grazier's and Robutel's papers due to different timescales. Their work focused on research of long time stability of Solar System regions while we studied the paths of a small ensemble of orbits. We believe that the results of our experiment and Robutel's survey would be similar for the same timescales of integrations.

Further studies are required to broaden our knowledge on orbital stabilization by overlapping and many-body MMRs in the chaotic region of the Solar System.

Acknowledgements. We thank G. Sitarski for help and comments. Many thanks to the referee F. Roig for his valuable discussions and helpful reviews. The computations were accomplished in the Poznań Supercomputing and Network Centre and in the Interdisciplinary Centre of Mathematical Modelling of the Warsaw University.

\section{References}

Bielicki, M., \& Sitarski, G. 1991, Acta Astron., 41, 309

Dvorak, R., Contopoulos, G., Efthymiopoulos, Ch., \& Voglis, N. 1998, Planet. Space Sci., 46, 1567

Everhart, E. 1973, AJ, 78, 316

Everhart, E. 1974, CM\&DA, 10, 35

Everhart, E. 1985, Dynamics of Comets: Their Origin and Evolution, ed. A. Carusi, \& G. B. Valsecchi, 185

Franklin, F., Lecar, M., \& Soper, P. 1989, Icarus, 79, 223

Grazier, K. R., Newman, W. I., Varadi, F., Kaula, W. M., \& Hyman J. M. 1999, Icarus, 140, 353

Holman, M. J. 1997, Nature, 387, 785

Kirkwood, D. 1867, Meteoric Astronomy, 105-111, ed. J. B. Lippincott (Philadelphia)

Levison, H. F., \& Duncan, M. J. 1997, Icarus, 127, 13

Milani, A., Carpino, M., Hahn, G., \& Nobili, A. M. 1989, Icarus, 78, 212

Milani, A., \& Baccili, S. 1999, CM\&DA, 71, 35

Nesvorny, D., \& Roig, F. 2000, Icarus, 148, 282

Robutel, P., \& Laskar, J. 2001, Icarus, 152, 4

Roig, F., Nesvorny, D., \& Ferraz-Mello, S. 2002, MNRAS, 335, 417

Sussman, G. J., \& Wisdom, J. 1988, Science, 241, 433

Sussman, G. J., \& Wisdom, J. 1992, Science, 257, 56

Sitarski, G. 1971, Acta Astron., 21, 87

Sitarski, G. 1979, Acta Astron., 29, 401

Sitarski, G. 1984, Acta Astron., 34, 53

Sitarski, G. 1998, Acta Astron., 48, 547

Tsiganis, K., Varvoglis, H., \& Hadjidemetriou, J. D. 2000, Icarus, 146, 240 